\title{
Construction of a Demand and Capacity Model for Intensive Care and Hospital Ward Beds, and Mortality From Covid-19
}

Christopher Martin ( $\nabla$ chris.martin@crystallise.com )

Crystallise Ltd

\section{Stuart McDonald}

Lloyds Banking Group (United Kingdom)

\section{Steve Bale}

Munich Re UK Life Branch

Michiel Luteijn

Hannover Re UK Life Branch

\section{Rahul Sarkar}

Medway NHS Foundation Trust

\section{Research Article}

Keywords: COVID-19, demand, capacity, model, mortality, public health, infectious diseases

Posted Date: March 6th, 2021

DOI: https://doi.org/10.21203/rs.3.rs-274009/v1

License: (9) This work is licensed under a Creative Commons Attribution 4.0 International License. Read Full License 
1 Title page

2 Construction of a demand and capacity

3 model for intensive care and hospital ward

4 beds, and mortality from COVID-19

5 Christopher Martin ${ }^{1,2}$, Stuart McDonald $^{3}$, Steve Bale ${ }^{4}$, Michiel Luteijn $^{5}$, Rahul Sarkar $^{6}$,

$6 \quad *$ Correspondence: chris.martin@crystallise.com

$7 \quad{ }^{1}$ Director of Modelling at Crystallise, Unit 19, Saffron Court, Southfields Business Park, Basildon, Essex,

8 SS15 6SS.

9 Abstract

10 Background

11 This paper describes a model for estimating COVID-19 related excess deaths that are a direct

12 consequence of insufficient hospital ward bed and intensive care unit (ICU) capacity.

13 Methods

14 Compartmental models were used to estimate deaths under different combinations of ICU and ward

15 care required and received in England up to late April 2021. Model parameters were sourced from

16 publicly available government information and organisations collating COVID-19 data. A sub-model was 
17 used to estimate the mortality scalars that represent increased mortality due to insufficient ICU or

18 general ward bed capacity. Three illustrative scenarios for admissions numbers, 'Optimistic', 'Middling' and 'Pessimistic', were modelled and compared with the subsequent observations to the $3^{\text {rd }}$ February.

20 Results

21 The key output was the demand and capacity model described. There were no excess deaths from a lack

22 of capacity in the 'Optimistic' scenario.

23 Several of the 'Middling' scenario applications resulted in excess deaths - up to 597 deaths (0.6\%

24 increase) with a $20 \%$ reduction compared to best estimate ICU capacity.

25 All the 'Pessimistic' scenario applications resulted in excess deaths, ranging from 49,178 (17.0\%

26 increase) for a $20 \%$ increase in ward bed availability, to 103,735 ( $35.8 \%$ increase) for a $20 \%$ shortfall in

27 ward bed availability. These scenarios took no account of the emergence of the new, more

28 transmissible, variant of concern (b.1.1.7).

\section{Conclusions}

30 Mortality is increased when hospital demand exceeds available capacity.

31 No excess deaths from breaching capacity would be expected under the 'Optimistic' scenario. The

32 'Middling' scenario could result in some excess deaths - up to a $0.7 \%$ increase relative to the total

33 number of deaths. The 'Pessimistic' scenario would have resulted in significant excess deaths. Our

34 sensitivity analysis indicated a range between 49,178 (17\% increase) and 103,735 (35.8\% increase).

35 Given the new variant, the pessimistic scenario appeared increasingly likely and could have resulted in a 36 substantial increase in the number of COVID-19 deaths. In the event, it would appear that capacity was 
37 not breached at any stage at a national level with no excess deaths. it will remain unclear if minor local

38 capacity breaches resulted in any small number of excess deaths.

\section{Keywords}

40 COVID-19, demand, capacity, model, mortality, public health, infectious diseases

\section{Background}

42 The number of COVID-19 deaths in the UK was 74,125 deaths and the number of known cases was

$432,542,069$ as of the $2^{\text {nd }}$ January 2021 representing a case fatality rate (CFR) of $2.9 \%$. The Office for

44 National Statistics (ONS) Coronavirus (COVID-19) Infection Survey estimated that $8.7 \%$ of people in

45 England still had antibodies at detectable levels based upon serological testing.(1) Assuming that, as of 46 the $2^{\text {nd }}$ January 2021 , around $10 \%$ to $15 \%$ of the population of England has been infected (taking into

47 account those with antibodies no longer at detectable levels, the lag time and the small proportion of

48 false negative serology), then this would suggest that between 6.7 million and 10 million people had

49 already been infected representing an infection fatality rate (IFR) of $0.7 \%$ to $1.1 \%$.

50 IFR estimates are typically made in the context of adequate capacity of health care services including

51 hospital beds and ICU beds. Should the demand for ICU beds exceed the supply, then the IFR would be

52 expected to rise. In this paper we describe a demand and capacity model designed to estimate the

53 number of COVID-19 deaths that would directly arise from a lack of ICU and ward beds. Such a model

54 currently does not exist for the UK in the public domain.

55 In November 2020 we began development of a model to estimate what increases in deaths could be

56 expected as a direct consequence of a lack of hospital bed capacity, and in particular ICU beds. Due to 57 availability of data, the model was restricted to England. 
58 Estimating the ICU capacity in England is complicated, as the actual capacity at a given point of time

59 varies between usual bedbase and the maximum surge capacity created to handle increasing demand

60 during a pandemic. There were estimated to be 4,114 ICU beds in England pre-pandemic.(2) However,

61 there are plans in place to allow surges in ICU capacity when pandemics occur which entails repurposing

62 other hospital resources including anaesthetic rooms, operating theatres and parts of accident and

63 emergency departments. The aim was to be able to increase ICU bed capacity across the country by

$64100 \%$ in the event of a pandemic.(3) It is likely that the increase in capacity will vary by institution and

65 one case study managed to increase capacity by $236 \%$ during the first wave.(4) In the event of a

66 pandemic, the NHS is expected to respond through a whole system approach, which has been outlined

67 in the UK CRITCON scoring system. The basis for the system is that the same non-pandemic ethical

68 standards are applied to treat patients and allocate resources, unless in the extreme scenario (CRITCON

69 4). The system was formulated in 2009 for $\mathrm{H} 1 \mathrm{~N} 1$ and has been updated in 2020 in the context of the

70 COVID-19 pandemic. The levels described above extend from a normal capacity at CRITCON 0 to

71 CRITCON 4 when the system is overwhelmed. A "mutual aid" system exists to facilitate inter-hospital or

72 regional transfer of patients when the local intensive care capacity is breached in order to support the

73 principle that no patient should be deprived of the appropriate care if there is systemwide capacity

74 available. Eighteen intensive care networks in the England and Northern Ireland manage the capacity

75 and allocation to intensive care in the event of increasing demand.

76 At the start of the pandemic a series of field hospitals were constructed at eight sites across England.

77 The capacity provided by these field hospitals is flexible according to circumstance, but it is estimated

78 that this would potentially provide an additional 8,000 general ward beds and 500 ICU beds though

79 there may be staffing constraints that limit this number.(2) 
80 In this paper we describe a model for estimating the number of additional deaths that occur from a lack

81 of capacity under different scenarios. These are illustrative scenarios and not forecasts.

82 Parameterization of the model can be modified according to available information and updated over

83 time.

84 Methods

85 Model description

86 The model can be found on GitHub: https://github.com/Crystallize/COVID19 ExceedingCapacityModel.

87 The model is a series of static compartmental models that estimates the number of COVID-19 related

88 deaths in England up to late April 2021 under three conditions: availability of both general ward and ICU

89 care; availability of general ward care but no ICU care; and no availability of either general ward or ICU

90 care.

91 The model was developed as an Excel workbook as Excel allows rapid iteration and development with

92 transparency for other developers and reviewers as it is a widely available platform.

\section{General outline}

94 The general outline of the model is shown in Figure 1 Outline of the structure of the model. It operates

95 using a scenario of COVID-19 hospital admission demand in weekly time steps.

96 First, we modelled the expected age distribution of admissions. This is an important input as hospital

97 and intensive care admission rates and COVID-19 mortality vary substantially by age. Subsequently, the

98 weekly demand for intensive care and ward beds was modelled by age. The weekly availability of beds

99 was calculated using estimates of the number of free beds usually available in ICUs in England plus any 
100 surge capacity and additional capacity freed by the cancellation of routine surgery, field hospitals and

101 the use of private facilities adjusted for the average duration of stay on ICU and in the ward in general.

102 We then compared the weekly demand for ward and ICU beds with the maximum number of beds

103 available. This allowed for the number of weekly admissions that fall within or outside of capacity.

104 Next, we calculated an estimate of the multiplier of the mortality rates when an ICU bed is not available

105 to someone who needs it, and similarly for general ward care. This was done by compartmentalizing

106 ward and ICU patients to categories of care for which estimates were made and then aggregated up to

107 the ward or ICU level again. The assumption is that the ICU mortality rate is multiplied by 1.99 if there is

108 no ICU bed available, but there is a ward bed, and by 9.02 if there is neither a ward not ICU bed

109 available. For general ward patients, the multiplier is 3.69 if there are no ward beds available. More

110 detail on the derivation of this is provided in the section on the "Excess Mortality Model". Sensitivity

111 testing indicated that results were not especially sensitive to small changes in these mortality multipliers

$112(+/-20 \%)$.

113 Once COVID-19 mortality rates for ward and ICU patients both within and outside of capacity were

114 calibrated, the number of deaths occurring 'within capacity' and the number that occur directly as a

115 result of being out of capacity could be calculated. The number of 'out of capacity' deaths does not

116 include the number of deaths that would have been expected to occur if normal care had been received,

117 and so represent 'excess' deaths occurring because the capacity limit was breached.

119 We will now describe the purpose and workings of the model in more detail. 
121 The pre-pandemic (i.e., before March 2020) spare bed availability in the general wards in England is 122 estimated at 9,769 and the ICU spare bed capacity as 817 beds from a study of hospital capacity in the 123 COVID-19 pandemic by the Medical Research Council, Public Health England, The National Institute of 124 Health Research and Imperial College.(2) The same study estimated an additional 1,810 ICU beds and 12552,498 beds could be acquired using field hospitals, the cancellation of routine care and the use of 126 private hospital facilities. In addition, there are pandemic response plans in place to increase ICU 127 capacity with a surge in demand. This surge capacity would be intended to increase ICU beds by $100 \%$ by 128 using anaesthetic rooms, operating theatres and other hospital resources.(3) Altogether this would 129 increase spare ICU capacity to 3,444 beds and spare ward capacity to 62,267 beds. These 62,267 ward 130 beds can provide for 87,174 patients weekly. These figures are based on an estimated length of stay on 131 ICU of 7 days and length of stay on the general ward of 5 days. $(5,6)$

[AdditionalFile_1_Figure_1_ModelStructure.pptx].

133 Figure 1 Outline of the structure of the model.

134 Estimating COVID-19 Admissions by Age

135 The age distribution of admissions was required in 5-year age bands up to the age of 80 years with one 136 category for those of age 80-years and over (if this data is available it can be applied directly in the 137 model removing the need for this estimation step). The age distribution in ICUs is lower than on the 138 wards with an average age of 62 years and with only a small proportion of those over 80 -years being fit 139 enough for invasive ventilation.(5) However, we estimated the distribution of admissions for each age140 band for each calendar week by fitting an exponential curve to age-binned data on admission rates for 141 adults, then interpolating admission rates for each year of age. Using population estimates from 2019 
142 for each year of age, estimates of the numbers of admissions were made.(7) These were then

143 recalibrated so that the exact number of admissions in each age group matched the observed numbers

144 in each corresponding age-band.

145 New COVID-19 patients in hospital

146 In this step, we applied the distribution of admissions by age to the weekly admission demand from the

147 scenarios to calculate the percentage of admissions accounted for by each year of age.

148 Splitting COVID-19 Admissions into ICU and Ward

149 Next, we calculated the proportion of admissions that require intensive care and ward care by age for

150 each 5-year age band, with one age band for those aged 80 years or over. This is done using data on the

151 age breakdown of admissions and total admission numbers to ICU from the ICNARC report of the $18^{\text {th }}$

152 December 2020, and the total number of COVID-19 hospital admissions up to the $18^{\text {th }}$ December 2020

153 from the Gov.UK COVID-19 data dashboard.(8,9)

\section{COVID-19 Ward Demand}

155 In this step, we separated the weekly demand for general ward care by age into two tables; one for

156 those cared for within the expected hospital capacity, and a second for those who fail to receive any

157 hospital care when required because of lack of capacity.

158 COVID-19 ICU Demand

159 In this step we separated the weekly demand for ICU care by age into three tables; one for those cared 160 for within the expected ICU capacity, a second for those who receive only ward care when ICU care is 161 required because of no capacity, and a third for those who fail to receive any hospital care when 162 required. 
164 This is a critical step in the modelling as it is here that the estimate of the increase in COVID-19 mortality in the absence of a ward or ITU bed is determined. It would be very challenging to directly make a meaningful estimate of the increase in mortality overall, given the diverse range of severity of COVID-19 and the distribution of people with COVID-19 across the different pathways that unfold. In order to reduce uncertainty, it is necessary to map the flow of individuals through treatment pathways and consider the impact on mortality that would arise from depriving any individual requiring that element of care. The uncertainty in estimating the impact of lack of capacity is greatly reduced by considering each element of care in the pathway

170 separately than when considering the impact across the pathway as a whole. Consequently, we have taken the approach of

171 compartmentalizing the care-pathway for COVID-19, identifying the most critical element of the pathway for different

172 individuals and the volume of people in those compartments from observed data. For example, for people who never need more

173 than high-flow oxygen on a general ward (>35\% O2), they are allocated to this compartment and their risk is not related to

174 lower-risk care compartments like 'general ward care'. Similarly, for individuals who pass through general ward care and high

175 flow oxygen to ITU admission, continuous positive airways pressure' support (CPAP) and eventually invasive mechanical

176 ventilation (IMV), then they are allocated to riskiest of these compartments - the IMV. In the latter case, we can observe the

177 survival from data in this group and also make a reasonably reliable judgment that if the option of IMV is removed, then the

178 survival will be close to zero. There will be greater uncertainty in the estimation of the impact of removing lower levels of care,

179 but structure can be applied to estimating the impact of depriving this care by considering the fatal events that are averted by

180 the care and their risk in the absence of that care. The evidence and assumptions used in setting the mortality rates in each

181 compartment can be found in

Table 2. All required judgements were made by $\mathrm{CM}$, a former clinician and the principal architect of this sub-model in conjunction with RS, a physician in respiratory medicine and critical care.

184 We estimated the multiplier of COVID-19 mortality risk for ward and ICU care COVID-19 patients when there is 1) no ICU capacity and 2) when there is neither ward nor ICU capacity in comparison to the in- 
188 The proportion of patients requiring intensive care was taken from the results of the modelling step 189 "Splitting Admissions into ICU and Ward" (8\%). Those receiving general ward care or ICU care were 190 segmented into the categories shown in Error! Reference source not found.. The distribution of ward 191 care patients across the care categories was populated using observation data from the Nottingham 192 Universities Hospitals Trust.(10) The proportion of ICU patients only requiring supportive care or high 193 flow oxygen was also take from the Nottingham data. The rest of the ICU categories were populated in 194 line with published data from University Hospital Southampton for similar group of patients.(11) 
Table 1. Hospital care compartments, proportion occupancy and the mortality rates under three capacity scenarios.

\begin{tabular}{|c|c|c|c|c|c|}
\hline Proportion & Care segment & $\begin{array}{l}\text { Proportion in } \\
\text { each group }\end{array}$ & $\begin{array}{l}\text { COVID-19 } \\
\text { Mortality: } \\
\text { in capacity }\end{array}$ & $\begin{array}{c}\text { COVID-19 } \\
\text { Mortality: no } \\
\text { ICU }\end{array}$ & $\begin{array}{l}\text { COVID-19 } \\
\text { Mortality: } \\
\text { no ward or } \\
\text { ICU }\end{array}$ \\
\hline \multirow{3}{*}{$92 \%$} & General ward care & 0.6 & 0.01 & 0.01 & 0.03 \\
\hline & Ward care: $\mathrm{O}_{2}>35 \%$ & 0.17 & 0.01 & 0.01 & 0.5 \\
\hline & $\begin{array}{c}\text { Ward care: } \mathrm{O}_{2}>35 \% \\
\text { (Ceiling) }\end{array}$ & 0.23 & 0.4 & 0.4 & 0.95 \\
\hline \multirow{4}{*}{$8 \%$} & ICU: Supportive/HFO & 0.48 & 0.01 & 0.02 & 0.75 \\
\hline & ICU: NIV/CPAP & 0.16 & 0.01 & 0.02 & 0.95 \\
\hline & $\begin{array}{l}\text { ICU: NIV/CPAP } \\
\text { (Ceiling) }\end{array}$ & 0.12 & 0.83 & 0.9 & 1 \\
\hline & ICU: IMV+/-ECMO & 0.24 & 0.4 & 1 & 1 \\
\hline
\end{tabular}

198 non-invasive ventilation, CPAP - continuous positive airways pressure, IMV - invasive mechanical 199 ventilation, ECMO - extracorporeal membrane oxygenation, Ceiling - this refers to the most intensive 200 level of care that is appropriate to each patient depending on their level of frailty and usually 201 determined by the Clinical Frailty Score (CFS).

202 The expected numbers of deaths in each category calculated using the assumed mortality rate and 203 weighted by the proportions in the categories using the formulae below. 


$$
q=\frac{\sum_{c=1}^{c=n} \text { death }_{c}}{\operatorname{hosp}}
$$

205 Where:

$206 q=$ probability of dying in this episode of COVID-19

207 deaths $_{c}=$ the number of deaths in the care category ' $\mathrm{c}$ '

$208 h o s p=$ the number of people hospitalised with COVID-19

$$
m=-(1-L N(1-q))
$$

210 Where:

$211 m=$ hazard rate for death corresponding to the probability of dying in the scenario ' $q$ ', and

$$
H R=\frac{m_{s}}{m_{b}}
$$

213 where $H R=$ hazard ratio applied to the base-case mortality rate $m_{b}$ to find the mortality rate in

214 scenario 's'.

215 From the Excess Mortality Sub-model, we estimated a hazard ratio of 1.99 for COVID-19 mortality in

216 those needing ICU care when no ICU bed was available but there was a ward bed, and 9.02 when there

217 was neither an ICU nor ward bed available. For those needing ward care only, the hazard ratio is 3.69

218 when no hospital bed is available. Sensitivity testing indicated that results were not especially sensitive

219 to small changes in these hazard ratios (+/- 20\%).

220 Ward Mortality - Treated

221 In this step, we estimated the in-capacity COVID-19 mortality rate by age in 5-year age bands

222 interpolating from data from the cumulative COVID-19 daily deaths report on the NHS England website 
223 and using the age distributions for admissions calculated in the modelling step "Estimating Admissions

224 by Age".(12) Data was harvested from the $6^{\text {th }}$ November 2020 dataset as this precedes the peak of the

225 second wave when pressure may already have been building on internal hospital resources. After

226 estimating the mid-points of the age-bands, an exponential model is fitted to the mortality rates for the

227 three age bands from age 40 upwards. This fitted model is then used to interpolate the mortality rates

228 for the 5-year age bands required.

229 ICU Mortality - Treated

230 In this step, we calculated the in-capacity COVID-19 mortality rates by 5-year age bands in ICU. Data was

231 taken from the $6^{\text {th }}$ November 2020 ICNARC report on COVID-19 in intensive care.(13) The mortality rate

232 for each age band was calculated from the sum of the product of the number of admissions for that age

233 band, the 28-day in-hospital mortality rate and the total number of admissions for that age band. An

234 exponential model was then fitted to the data to which age bands had been applied in order to allow

235 interpolation and re-categorising by 5-year age-bands.

236 COVID-19 Mortality Rates

237 In this step we took the COVID-19 mortality rates by age calculated in the previous two modelling steps

238 and the hazard ratios from the 'Excess Mortality Model' step to calculate the mortality rates when no

239 ICU beds and no ward beds are available respectively. The mortality rate under the scenario ' $s$ ' is

240 calculated using the equation:

$$
q_{s}=1-(1-q)^{H R_{S}}
$$

242 Where:

$243 q=$ the mortality rate in the in-capacity scenario.

$244 H R_{S}=$ the hazard ratio in scenario 's' 
$245 q_{s}=$ the mortality rate in the scenario 's'

246

247 Table 2 Expert judgements made on mortality rates by care category in the excess mortality sub-model.

248 [Note to authors - this table is supplied as a separate file

249 'BMC_IFOA_COVID_1_AdditionalFile_2_Table_2_AssumptionsExcessMortality.pptx' as it is too large to

250 fit here.]

251 Legend for Table 2: ICU - intensive care unit, HFO - high flow oxygen, NIV - non-invasive ventilation,

252 CPAP - continuous positive airways pressure, IMV - invasive mechanical ventilation, ECMO -

253 extracorporeal membrane oxygenation, CFS - clinical frailty score, Ceiling - this refers to the most

254 intensive level of care that is appropriate to each patient depending on their level of frailty and usually

255 determined by the Clinical Frailty Score (CFS).

256 COVID-19 Deaths - In capacity

257 In this step, we calculated the number of COVID-19 deaths each week from the corresponding number 258 of admissions generated by the 'Ward Demand' and 'ICU Demand' steps and the in-capacity mortality 259 rates from the previous 'Mortality Rates' step. Observed data is used up to the $9^{\text {th }}$ December 2020, with 260 ICU deaths calculated as $15 \%$ of the total number of deaths (as there are significant delays in reporting 261 the deaths in the ICNARC data).

262 Deaths-Outside capacity

263 In this step, we calculated the numbers of deaths arising each week as a direct result of failing to get an 264 ICU or ward bed. Observed data is used up to the $9^{\text {th }}$ December 2020. A series of seven calculations were 265 used: 
1. The number of deaths in ICU within capacity as the dot product of the vector of ICU demand within ICU capacity by age from step 'ICU Demand' and the vector of mortality rates from step 'Mortality Rates'.

2. The number of deaths in patients requiring ICU care where only ward care was available, as the dot product of the vector of ICU demand outside ICU by age from step 'ICU Demand' and the vector of mortality rates from step 'Mortality Rates'.

272 3. The number of deaths in patients requiring ICU care where neither ward nor ICU care was available as the dot product of the vector of ICU demand outside both ICU and ward by age from step 'ICU Demand' and the vector of mortality rates from step 'Mortality Rates'.

4. The number of deaths in patients requiring ward care only as the dot product of the vector of ward demand within ward capacity by age from step 'Ward Demand' and the vector of mortality rates from step 'Mortality Rates'. dot product of the vector of ward demand outside ward capacity by age from step 'Ward Demand' and the vector of mortality rates from step 'Mortality Rates'.

6. The total number of deaths each week under the current scenario including both ICU and ward deaths as the sum of all results in steps 1-5.

7. The total number of deaths in each calendar week in both the ICU and ward care groups calculated in the step 'Deaths - In capacity' was taken and subtracted from the capacity constrained total in step 6 to provide the number of deaths arising as a direct result of the lack of an ICU or ward bed. 


\section{Scenarios}

288 We applied three scenarios for admission demand from the week beginning $16^{\text {th }}$ December 2020

289 through to the week beginning $28^{\text {th }}$ April 2021 (see Figure 2).

290 [AdditionalFile_3_Figure_2_AdmissionsDemandScenario).

291 In this model, the three scenarios are referred to as "Pessimistic", "Middling" and "Optimistic" and were

292 arbitrary but based on the trajectory of the increase in the multiplier of admissions from one week to

293 the next. The trajectory was calculated as a linear regression of the last three weeks of the known data

294 taken from the GOV.UK Coronavirus dashboard.(8) They are illustrative scenarios and not forecasts.

295 With the passage of time, further data through to the week of the $3^{\text {rd }}$ of February was obtained for 296 comparison with the scenarios. The peak in admissions actually occurred around the week of the $13^{\text {th }}$ of 297 January.

298 In the Middling scenario, the multiplier continued to increase following the rate over the previous 3 299 weeks up to three weeks post the introduction of more vigorous countermeasures on $19^{\text {th }}$ December 300 2020. This is to be expected as the incubation period is nearly a week, so the enhanced

301 countermeasures would usually take at least a week to result in any observed change. The multiplier

302 falls below 1.0 after 4 more weeks. The emergence of a new variant of SARS-CoV-2 with higher

303 transmission rates that became more prevalent from the beginning of December has driven more rapid

304 growth of infection rates. As the new variant makes up a greater proportion of new cases, the rate of

305 growth will continue to rise unless effectively mitigated. It remains uncertain, at the time of writing, how

306 it will respond to tighter controls, but there is no reason to assume the mortality rates will differ at

307 present. This new variant has only recently been identified and has not been factored into the analysis.

308 However, initial indications are that this variant could open up a variety of more pessimistic scenarios in 309 the short term. 
310 We now have effective vaccines and it is expected that their use will prevent a large number of

311 infections and many deaths (14).

312 In the Optimistic scenario, the multiplier begins to decelerate from week beginning $16^{\text {th }}$ December,

313 continues to decelerate after the $16^{\text {th }}$ December and falls below 1.0 after 3 weeks.

314 In the Pessimistic scenario, the multiplier continued to increase for 4 weeks after week beginning $16^{\text {th }}$

315 December before decelerating at the same rate as the other two scenarios. This is not intended as a

316 worst-case scenario, but one that is somewhat worse than the 'Middling' scenario.

317 Figure 2 Graph showing three scenarios of projected admission demand from the 9th December 2020.

\section{Sensitivity analysis}

319 We performed an analysis to assess the sensitivity of the model to the choice of capacity and hazard

320 ratio parameter values and scenario. Five input parameters were varied independently in turn and the

321 effect on the model output recorded. The five parameters varied were the ICU and ward capacities and

322 the hazard ratios for mortality in ward patients when there are no ward beds available and ICU patients

323 when there are no ICU beds and ICU patients when there are no ward beds available. The hazard ratios

324 were adjusted using the equation.

$325 H R^{*}=1+(H R-1) \cdot(1+d)$

326 Where:

$327 \quad H R=$ the within-capacity hazard ratio.

$328 H R^{*}=$ the outside-capacity hazard ratio.

$329 d=$ the adjustment to be applied as a proportion. 
330 This model has been populated with data specific to England, it could be applied in other geographies

331 and we have added ways of estimating some data like the age distribution of admissions or the ICU

332 admission rates by age. Where this data is directly available it can be substituted.

\section{Assumptions}

334 There are several general underlying assumptions to the model.

- There is no change in the expected mortality as demand grows until the bed capacity is breached. capacity may be a product of all of the above.

- Only bed capacity affects excess mortality rates and not other resource constraints such as staffing, equipment, ambulance availability or other finite resources, although actual system

- Health care policy and conscious or unconscious clinician behaviour that may affect the compartments of care through which the patients flow does not change as the pandemic waxes and wanes.

- The age distribution of admissions remains constant over time.

- There is perfect redistribution of bed capacity and resources across the country immediately according to demand.

- Weekly bed availability remains constant.

- Capacity estimates are fixed throughout the model when, in fact, new resources may be recruited over time, or capacity may fall in response to disruption of staffing and supplies. 
- The mortality rates, including by age, are the same for the old COVID strain and novel mutations.

\section{Results}

356 The key output of our collaboration was the model itself rather than the results of any of the scenarios.

357 The model allows a user to understand the excess COVID-19 mortality impact arising as a direct

358 consequence of ward and/or ICU capacity being breached under various scenarios or forecasts of

359 hospital admissions. The scenarios described in this paper are illustrative and are not forecasts.

360 Table 3. Numbers of deaths with the unadjusted middling scenario from $16^{\text {th }}$ of December (bold font).

361 [Insert Table 3 here - Table 3 at end of file as per BMC guidelines for tables larger than 1A4 in length]

\section{Sensitivity analyses}

363 The results of the sensitivity analysis are shown in Table 4 and Figure 3. None of the Optimistic scenario

364 adjustments resulted in any excess deaths. In the Middling scenario adjustments, the excess deaths

365 attributable to lack of capacity alone ranged from 0 to 597 with a $20 \%$ reduction in the expected number

366 of ICU beds. In the Pessimistic scenario the number of excess deaths attributable directly to lack of

367 capacity range from 49,178 with a $20 \%$ increase in the number of ward beds, and 103,735 with a $20 \%$

368 reduction in the expected ward bed availability.

369 Figure 3. Results of the sensitivity analysis.

370 [Additional File 4_Figure_3_SensitivityAnalysis]

371 The results can be explained by considering how capacity evolves in each of the scenarios. In the

372 Middling scenario, whilst ICU capacity may be approached and even possibly breached, there remains

373 sufficient ward capacity to take lives who need either ward or ICU support, keeping excess deaths 
374 relatively low. However, the Pessimistic scenario sees ward capacity breached, and in many scenarios

375 for a period of several weeks, resulting in much higher mortality in those lives who require care but do

376 not receive it. ICU capacity is much lower than ward capacity and only a small proportion of all

377 hospitalized patients need ICU care so the number of deaths from breaches of ward capacity are

378 proportionally larger than breaches for ICU care. ICU care is assumed to reach capacity before ward

379 care, so with marginal breaches of capacity, ICU breaches are the source of the excess deaths, but with

380 large breaches of capacity, the majority will arise from breaching of ward capacity.

381 The number of excess deaths is most sensitive to ward bed availability in the pessimistic scenario with a

382 difference of 54,556 excess deaths between the $20 \%$ increase and $20 \%$ decrease in the bed availability

383 compared to 5,129 with the same variation in ICU bed availability. However, in the Middling scenario,

384 the excess deaths are most sensitive to ICU bed capacity with a difference of 597 with +/- $20 \%$ variation

385 in the ICU bed capacity estimate, with no difference arising from the ward bed capacity estimate.

386 These results are shown graphically in Additional File 3_SensitivityAnalysis (note the different y-axis

387 scales). Excess deaths are more sensitive to the availability of ICU or ward beds than to the adjustments

388 in the hazard ratios used here. The greatest loss of life occurs with a $20 \%$ reduction in the estimate of

389 ward availability in the pessimistic scenario with 103,735 (35.8\% increase) excess deaths.

390 The actual observations including data from the $16^{\text {th }}$ December through to the week of the $3^{\text {rd }}$ of

391 February showed a peak weekly admission rate of 29,447 in the week of the $13^{\text {th }}$ of January before

392 declining (Figure 2). There would have been no excess deaths due to lack of capacity in the observed

393 cases through to February under this set of assumptions. 


\section{Discussion}

395 Table 4. Results of the sensitivity analysis.

396 [Insert Table 4 here - Table 4 at end of file as per BMC guidelines for tables larger than 1A4 in length]

398 The new variant of SARS-CoV-2 that emerged in the UK (B.1.1.7) appears to be about $56 \%(95 \% \mathrm{Cl} 50 \%$ -

$39974 \%)$ more transmissible than the existing variants and appears to have a higher case-fatality rate $(15,16)$

400 Alternative explanations for its rise in prevalence and the increased rate of transmission observed since

401 its appearance at the beginning of October were investigated including "immune escape" where

402 individuals previously infected return to susceptibility as a result of mutation of key antigens, increased

403 susceptibility amongst children, and a shorter generation time. None of these alternative explanations

404 fitted the data as well as increased infectiousness. In addition, a variant has emerged from South Africa

405 (501Y.V2) which also appears to have greater transmissibility and an increased viral load.(17) These

406 unsettling developments increases uncertainty in the future trajectory of hospital demand and open up

407 a variety of significantly more pessimistic scenarios in the short term that have not been possible to

408 explore here.

409 In the longer term, there are now three vaccines approved for use in the UK, as of the $19^{\text {th }}$ January 2021

410 (18). It is expected they will have a substantial impact on admissions and deaths, though not until after

411 the majority of deaths in our scenarios are modelled as occurring (19). Consequently, we have not

412 factored the application of vaccination into the scenarios as they are operating in the short-term.

\section{Limitations}

414 Here we list and discuss the limitations of the model: 
415 - The estimate of bed availability is determined using the mean length of stay on the ward or in

416 ICU. The distribution of occupants by length of stay will change over time which may result in a

417 slow consumption of capacity that is not captured in this model. For example, a higher

418 proportion of afflicted younger patients in hospital may lead to longer bed occupancy both in

419 ward and critical care, as withdrawal of active treatment in view of futility is less likely to take

$420 \quad$ place in this group.

421 - Mortality rates are affected by constraints other than just bed availability including staffing and

422 equipment.

423 - The model will not capture the transition between low mortality with full capacity and the high

424 mortality from lack of a bed that arises from stressing of the system before capacity is

425 absent.(20)

426 - Capacity estimates are fixed throughout the model when, in fact, new resources may be

427 recruited over time, or capacity may fall in response to disruption of staffing and supplies.

428 - Age specific case fatality rates are assumed to be static, but in fact may change, either due to

429 changes in virulence, improvement in care or dilution of standards of care during a surge

$430 \quad$ scenario.

431

- The oxygen cut-off of $35 \%$ is based on clinical opinion, and there is no empirical study in support of this threshold for obvious reasons. However, often clinical practice may be to err on the side of caution in a ward setting and to keep a patient on a slightly higher fractionated oxygen than s/he needs and therefore a patient on a lower than 35\% FiO2 may actually need even smaller amounts of oxygen, and therefore the assumption is that $s /$ he would withstand the lack of medical oxygen with a degree of success. 
438 Intensive care resources are constrained not only by bed availability but also by equipment and staffing.

439 A combination of all three is required for optimal care in ICU. In reality there is not a simple binary state

440 of presence or absence of these factors and skills. A degradation of equipment maintenance, training

441 around new equipment and distribution as well as reductions in the effectiveness of staff, either

442 because staff-to-patient ratios fall as demand rises, or because of staff sickness due to COVID-19 or

443 simply the physical and emotional fatigue as the pandemic continues is also seen. An analysis of within-

444 capacity mortality rates in ICU by bed occupancy has found that there is an almost linear increase in

445 mortality with no excess mortality at $0 \%$ bed occupancy to a $92 \%$ increase in mortality by $100 \%$

446 occupancy.(20)

447 The modelling by McCabe et al suggests that ICU capacity is first constrained by bed availability, though

448 lack of nurses and junior doctors is close behind.(2) They included sickness absence rates taken from

449 surveys of union members suggesting $15 \%$ of doctors were off sick in the first wave and may under-

450 estimate the impact of sickness on staffing overall at peak times in the pandemic.(21) As the pandemic

451 progresses, higher than anticipated absence due to sickness in these groups could result in lack of

452 limiting capacity before the lack of beds, both in ICU and in wards. Reorganisation within hospitals may

453 mitigate this by training other staff to support ICU work and thereby increasing ICU staff to bed

454 ratios.(22) One hospital managed to meet demand in the first wave, but reduced ICU nurse to bed ratios

455 from the normal pre-pandemic of 1:1 to 1:4.(4) Current guidance on nursing staff ratios during the

456 COVID-19 pandemic advocates a ratio of 1:2.(22)

457 Decision making may change in the face of increasing demand either with formal revisions of treatment 458 thresholds as resources become increasingly scarce or with the introduction of triaging. On a less formal 459 level, the heuristics used by clinicians in their everyday management of patients may vary as competing 460 pressures rise. For example, at times of abundant capacity, the thresholds for transferring patients into 
461 ICU for a trial to see if a patient with poor chance of survival recovers with a short stay, may be lower 462 than when there is severe limitation on capacity.

463 One of the most uncertain parts of the modelling is the determination of the multipliers of risk in the 464 section 'Excess Mortality Submodel'. We estimated that the multiplier for the scenario of no ICU bed 465 when one was required was 1.99. A recent observational study of 4,032 ICU admissions across 114 466 hospital trusts found a rising mortality with bed occupancy (20). The estimated excess mortality rate was 467 estimated to reach $92 \%$ at $100 \%$ capacity. This is very close to our estimate of $99 \%$ and is a reassuring 468 triangulation of the method.

469 An important but unrealistic assumption in the model is that there is perfect distribution of resources 470 with respect to demand across the country and that no patient is refused care whilst there remains a 471 bed anywhere in England. ICUs are organized into networks which facilitate the transfer of patients from 472 one hospital to another when ICU beds in a hospital run out, but even a delay of a few hours in 473 transferring a patient to an ICU bed can influence outcome. Furthermore, even in the case of bed 474 availability in a different hospital, it cannot be assumed that the patient in the referring and at-capacity 475 hospital may be fit enough to able to be transferred safely. All the ICUs in a network are likely to have 476 correlated demand and may all run out of space at the same time. In these circumstances, transfers 477 would need to be arranged between networks or regions and this would entail yet further delay and 478 challenges with consequent impact on outcomes.

479 It is notable that the sensitivity of the excess deaths to ICU capacity is much greater than it is to ward 480 bed capacity at marginal breaches of capacity, but that large scale breaches of capacity are more 481 sensitive to ward bed capacity. There are far more ward beds than ICU beds, and there is an assumption 482 that ICU capacity would be exhausted long before ward bed capacity. 


\section{Conclusions}

484 Here we describe a demand and capacity model for general hospital and intensive care beds in the 485 context of the COVID-19 pandemic in England. The model allows a user to understand the excess COVID-

48619 mortality impact arising as a direct consequence of capacity being breached under various scenarios

487 or forecasts of hospital admissions. The scenarios described in this paper are illustrative and are not 488 forecasts.

489 We estimated the number of excess COVID-19 deaths up to the end of April 2021 that would arise from 490 lack of ward and ICU capacity under different demand assumptions from December $16^{\text {th }}, 2020$. No

491 excess deaths from excess capacity would be expected under the 'Optimistic' assumptions of demand 492 but would reach between 49,178 and 103,735 under the 'Pessimistic' scenario. Without the new variant, 493 exceeding capacity for hospital and ICU beds was not the most likely outcome but given the new variant 494 it appeared more plausible and could have result in a substantial increase in the number of COVID-19 495 deaths. In the event, it would appear that capacity was not breached at a national level any stage, and, 496 under an assumption of a perfectly even distribution of demand and capacity, there would be no excess 497 deaths due to lack of capacity expected under this set of assumptions. However, distribution of demand 498 and capacity is imperfect. It will remain unclear if minor local capacity breaches resulted in any small 499 number of excess deaths.

\section{List of abbreviations}

501 CFR: Case fatality rate

502 CFS: Clinical frailty score

503 CPAP: Continuous positive airway pressure 
504 ECMO: Extracorporeal membrane oxygenation

505 HFO: High flow oxygen

506 HR: Hazard ratio

507 ICNARC: Intensive care national audit and research centre

508 ICU: Intensive care unit

509 IFR: Infection fatality rate

$510 \quad$ IMV: Invasive mechanical ventilation

511 NIV: Non-invasive ventilation

512 ONS: Office of national statistics

513 SARS-CoV-2: Severe acute respiratory syndrome coronavirus 2

514 VOC: Variant of concern

515 Declarations

516 Ethics approval and consent to participate

$517 \quad$ Not appliable

518 Consent for publication

519 Not applicable

520 Availability of data and materials

521 The dataset supporting the conclusions of this article is available GitHub:

522 Crystallize/COVID19 ExceedingCapacityModel (github.com). 


\section{Competing interests}

524 RS received writing fees for healthcare reports from Crystallise UK Ltd. None of the other authors 525 declare any conflict of interest.

526 Funding

527 No funding source.

528 Authors' contributions

529 Stuart McDonald initiated and coordinated the project and was one of the principal architects of the 530 general model and contributed to the final paper.

531 Chris Martin was the principal architect of the excess mortality sub-model, participated in the

532 development of the general model and pulled together the initial draft of the paper.

533 Michiel Luteijn participated in the development of the general model and undertook ancillary modelling

534 that supported development and contributed to the final paper.

535 Steve Bale participated in the development of the general model and contributed to the final paper.

536 Rahul Sarkar contributed to the expert judgements in the modelling, advised on the organizational

537 context within which the model sits, and contributed to the final paper.

538 Acknowledgements

539 Various

540 Colin Dutkiewicz, (Global Head of Life Reinsurance Solutions, Aon)

541 Josephine Robertson, (Health \& Care Actuary, Optum)

542 Dan Ryan, (Chief Science Officer at COIOS Research) 
543 Scott Reid, (Global Protection Pricing \& Product Development Actuary, Zurich)

544 James Robinson, (UK \& Ireland Business Strategy and Analytics, Aon Benfield)

$545 \quad$ Nottingham Universities Hospital Trust

546 Joe West, (Professor of Epidemiology; Honorary Consultant Gastroenterologist, Faculty of Medicine \&

547 Health Sciences Nottingham University)

548 Richard Hubbard, (GSK/British Lung Foundation Professor of Respiratory Epidemiology, Faculty of

549 Medicine \& Health Sciences)

550 Tim Card, (Clinical Associate Professor, Nottingham Biomedical Research Centre)

551 Colin Crooks, (Clinical Associate Professor, Nottingham Biomedical Research Centre)

552 Nina Lewis, (Consultant Gastroenterologist, Nottingham University NHS Trust)

553 Andrew Fogarty (Clinical Associate Professor \& Reader in Clinical Epidemiology, Faculty of Medicine \& 554 Health Sciences)

555 Andrew Marshall, (Deputy Medical Director at Nottingham University Hospitals)

556 Thearina De Beer, (Consultant in Anaesthetics and ICM at Nottingham University Hospitals NHS Trust)

557 Dominic Shaw, (Professor and Honorary Consultant, Faculty of Medicine \& Health Sciences)

558 Emma O'Dowd, (Honorary Consultant Assistant Professor, University of Nottingham)

559 Andrew Baraclough, (Assistant Director of Insight, Nottingham University Hospitals NHS Trust)

560 Crystallise

561 Shannon Connolly, Analysts at Crystallise Ltd. 
562 Dr Will Letton, Consultant Analyst at Crystallise

563 Dr Louisa Rutherford, Senior Clinical Researcher at Crystallise Ltd.

564 Additional files

565 https://github.com/Crystallize/COVID19 ExceedingCapacityModel.

566 Link to model on GitHub

567 BMC_IFOA_COVID_1_AdditionalFile_1Figure_1_ModelStructure.pptx

568 Title: "Figure 1. Outline of structure of model"

569 BMC_IFOA_COVID_1_AdditionalFile_2_Table_2_AssumptionsExcessMortality.pptx

570 Title: "Table 2. Expert judgements made on mortality rates by care category in the excess mortality sub-

571 model."

572 BMC_IFOA_COVID_1_AdditionalFile_3_Figure_2_AdmissionsDemandsScenarios.pptx

573 Title: "Figure 2. Graph showing three scenarios of projected admission demand from the $9^{\text {th }}$ December

$5742020^{\prime \prime}$

575 BMC_IFOA_COVID_1_AdditionalFile_4_Figure_3_SensitivityAnalysis.pptx

576 Title: "Figure 3. Results of the sensitivity analysis"

577 Author details

$578{ }^{1}$ Director of Modelling at Crystallise, Unit 19, Saffron Court, Southfields Business Park, Basildon, Essex, 579 SS15 6SS.

$580 \quad{ }^{2}$ Honorary Researcher, University College London. 
$581{ }^{3}$ Head of Demographic Assumptions and Methodology at Lloyds Banking Group, 25 Gresham Street,

582 London, EC2V 7HN

$583 \quad{ }^{4}$ Senior Actuary at Munich Re UK Life Branch, 10 Fenchurch Avenue, London EC3M 5BN

$584{ }^{5}$ Biometric Research Data Specialist at Hannover Re UK Life branch. 10 Fenchurch Street, London, EC3M

585 3BE.

$586{ }^{6}$ Consultant Physician in Respiratory Medicine \& Critical Care at Medway NHS Foundation Trust.

587 Windmill Road, Gillingham, Kent ME7 5NY

588

589 References

590 1. ONS. Coronavirus (COVID-19) Infection Survey: characteristics of people testing positive for

591 COVID-19 in England and antibody data for the UK: December 2020. Off Natl Stat.

592 2020;(December).

593 2. McCabe R, Schmit N, Christen P, D’Aeth JC, Løchen A, Rizmie D, et al. Adapting hospital capacity

594 to meet changing demands during the COVID-19 pandemic. BMC Med [Internet]. 2020 Dec

595 16;18(1):329. Available from: https://bmcmedicine.biomedcentral.com/articles/10.1186/s12916-

$596 \quad$ 020-01781-w

597 3. NHS England. Management of surge and escalation in critical care services : standard operating

598 procedure for adult critical care [Internet]. 2013 [cited 2020 Feb 15]. Available from:

599 https://www.england.nhs.uk/commissioning/wp-content/uploads/sites/12/2013/11/sop-

$600 \quad$ burns.pdf

601 4. Chong MSF, Hla TW, Sartori G. ICU surge capacity in a busy London district general hospital 602 during the COVID-19 pandemic [Internet]. Association of anaesthetists. 2020 [cited 2020 Dec 20]. 
603

604

605

606

607

608

609

610

611

612

613

614

615

616

617

618

619

620

621

622

623

624

Available from: https://anaesthetists.org/Home/Resources-publications/COVID-19-guidance/ICUsurge-capacity-in-a-busy-London-district-general-hospital-during-the-COVID-19-pandemic

5. ICNARC. ICNARC report on COVID-19 in critical care: England, Wales and Northern Ireland 24 December 2020 [Internet]. 2020. Available from: https://www.icnarc.org/OurAudit/Audits/Cmp/Reports

6. Rees EM, Nightingale ES, Jafari Y, Waterlow NR, Clifford S, Carl CA, et al. COVID-19 length of hospital stay: A systematic review and data synthesis. BMC Med. 2020;18(1).

7. Human Mortality Database [Internet]. 2020. Available from: https://www.mortality.org/

8. GOV.UK Coronavirus (COVID-19) in the UK [Internet]. 2020 [cited 2020 Dec 22]. Available from: https://coronavirus.data.gov.uk/

9. ICNARC. ICNARC report on COVID-19 in criӨcal care: England, Wales and Northern Ireland. 18 December 2020. 2020;(18th december). Available from:

file://C:/Users/ChrisMartin/AppData/Local/Temp/MicrosoftEdgeDownloads/9372fc02-31b041b4-9570-ff5bdb63fd1e/ICNARC_COVID-19_Report_2020-12-18.pdf.pdf

10. Crooks CJ, West J, Fogarty A, Morling JR, Grainge MJ, Gonem S, et al. Predicting the need for escalation of care or death from repeated daily clinical observations and laboratory results in patients with SARS-CoV-2 during 2020: a retrospective population-based cohort study from the United Kingdom. MedRxiv [Internet]. 2020; Available from:

https://www.medrxiv.org/content/10.1101/2020.12.14.20248181v1

11. Sivaloganathan AA, Nasim-Mohi M, Brown MM, Abdul N, Jackson A, Fletcher S V., et al. Noninvasive ventilation for COVID-19-associated acute hypoxaemic respiratory failure: experience from a single centre. $\mathrm{Br} J$ Anaesth. 2020;125(4):e368-71. 
625 12. NHS England. COVID 19 total announced deaths 23 December 2020 [Internet]. 2020 [cited 2020 626 Dec 23]. Available from: https://www.england.nhs.uk/statistics/statistical-work-areas/covid-19627 daily-deaths/

628 13. ICNARC. Report on COVID-19 in critical care. Intensive Care Natl Audit Res Cent [Internet]. 2020;(April):1-24. Available from: https://www.icnarc.org/About/LatestNews/2020/03/22/Report-On-196-Patients-Critically-III-With-Covid-19

14. Voysey M, Clemens SAC, Madhi SA, Weckx LY, Folegatti PM, Aley PK, et al. Safety and efficacy of the ChAdOx1 nCoV-19 vaccine (AZD1222) against SARS-CoV-2: an interim analysis of four randomised controlled trials in Brazil, South Africa, and the UK. Lancet [Internet]. 2021 Jan;397(10269):99-111. Available from: https://linkinghub.elsevier.com/retrieve/pii/S0140673620326611

15. Davies NG, Barnard RC, Jarvis Cl, Kucharski AJ, Munday J, Pearson CA, et al. Estimated 637 transmissibility and severity of novel SARS-CoV-2 Variant of Concern 202012/01 in England. Github [Internet]. 2020; Available from: https://cmmid.github.io/topics/covid19/reports/uknovel-variant/2020_12_23_Transmissibility_and_severity_of_VOC_202012_01_in_England.pdf

16. lacobucci G. Covid-19: New UK variant may be linked to increased death rate, early data indicate. BMJ [Internet]. 2021 Jan 26;n230. Available from: https://www.bmj.com/lookup/doi/10.1136/bmj.n230

17. SARS-CoV-2 Variants [Internet]. World Health Organisation. 2020 [cited 2021 Jan 4]. Available from: https://www.who.int/csr/don/31-december-2020-sars-cov2-variants/en/\#: :text=On 18 645 December\%2C national authorities, because of a N501Y mutation. 
647

648

649

650

651

652

653

654

655

656

657

658

659

660

661
2021 [cited 2021 Jan 19]. Available from: https://www.gov.uk/government/news/modernavaccine-becomes-third-covid-19-vaccine-approved-by-uk-regulator

19. Cook TM, Roberts J V. Impact of vaccination by priority group on UK deaths, hospital admissions and intensive care admissions from COVID-19. Anaesthesia [Internet]. 2021 Feb 11;anae.15442. Available from: https://onlinelibrary.wiley.com/doi/10.1111/anae.15442

20. Wilde H, Mellan T, Hawryluk I, Dennis J, Denaxas S, Pagel C, et al. The association between mechanical ventilator availability and mortality risk in intensive care patients with COVID-19: A national retrospective cohort study. MedRxiv [Internet]. 2021; Available from: https://www.medrxiv.org/content/medrxiv/early/2021/01/13/2021.01.11.21249461.full.pdf

21. Booth R. Number of NHS doctors off sick 'may be nearly triple the official estimate.' The Guardian. 2020.

22. NHS. Advice on acute sector workforce models during COVID-19 [Internet]. 2020. Available from: https://www.england.nhs.uk/coronavirus/wp-content/uploads/sites/52/2020/12/C0833_adviceon-acute-sector-workforce-models-during-COVID_with-apps_10dec.pdf

[Table 3 below] 


\begin{tabular}{|c|c|c|c|}
\hline Week & $\begin{array}{c}\text { Total COVID-19 Deaths } \\
\text { (with ICU and Ward capacity } \\
\text { limits) }\end{array}$ & $\begin{array}{l}\text { COVID-19 deaths if there are } \\
\text { no ICU and Ward capacity } \\
\text { limits. }\end{array}$ & $\begin{array}{l}\text { Excess COVID-19 deaths } \\
\text { specifically due to ICU and Ward } \\
\text { capacity limits }\end{array}$ \\
\hline $30 / 09 / 2020$ & 298 & 298 & 0 \\
\hline $07 / 10 / 2020$ & 444 & 444 & 0 \\
\hline $14 / 10 / 2020$ & 731 & 731 & 0 \\
\hline $21 / 10 / 2020$ & 1,074 & 1,074 & 0 \\
\hline $28 / 10 / 2020$ & 1,457 & 1,457 & 0 \\
\hline $04 / 11 / 2020$ & 1,784 & 1,784 & 0 \\
\hline $11 / 11 / 2020$ & 1,897 & 1,897 & 0 \\
\hline $18 / 11 / 2020$ & 2,103 & 2,103 & 0 \\
\hline $25 / 11 / 2020$ & 2,031 & 2,031 & 0 \\
\hline $02 / 12 / 2020$ & 1,889 & 1,889 & 0 \\
\hline $09 / 12 / 2020$ & 1,960 & 1,960 & 0 \\
\hline $16 / 12 / 2020$ & 2,685 & 2,685 & 0 \\
\hline $23 / 12 / 2020$ & 3,703 & 3,703 & 0 \\
\hline $30 / 12 / 2020$ & 5,697 & 5,697 & 0 \\
\hline $06 / 01 / 2021$ & 9,677 & 9,677 & 0 \\
\hline $13 / 01 / 2021$ & 11,689 & 11,612 & 77 \\
\hline $20 / 01 / 2021$ & 11,689 & 11,612 & 77 \\
\hline $27 / 01 / 2021$ & 9,289 & 9,289 & 0 \\
\hline $03 / 02 / 2021$ & 7,432 & 7,432 & 0 \\
\hline $10 / 02 / 2021$ & 5,945 & 5,945 & 0 \\
\hline
\end{tabular}




\begin{tabular}{|c|c|c|c|}
\hline $17 / 02 / 2021$ & 4,756 & 4,756 & 0 \\
\hline $24 / 02 / 2021$ & 3,805 & 3,805 & 0 \\
\hline $03 / 03 / 2021$ & 3,044 & 3,044 & 0 \\
\hline $10 / 03 / 2021$ & 2,435 & 2,435 & 0 \\
\hline $17 / 03 / 2021$ & 1,705 & 1,705 & 0 \\
\hline $24 / 03 / 2021$ & 1,193 & 1,193 & 0 \\
\hline $31 / 03 / 2021$ & 835 & 835 & 0 \\
\hline $07 / 04 / 2021$ & 585 & 585 & 0 \\
\hline $14 / 04 / 2021$ & 409 & 409 & 0 \\
\hline $21 / 04 / 2021$ & 286 & 286 & 0 \\
\hline $28 / 04 / 2021$ & 201 & 201 & 154 \\
\hline Total & 102,728 & 102,573 & \\
\hline
\end{tabular}

662

663 [Table 4 below]

\begin{tabular}{|c|c|c|c|c|c|c|c|c|}
\hline Scenario & $\begin{array}{c}\text { Ward } \\
\text { availability }\end{array}$ & $\begin{array}{c}\text { ICU } \\
\text { availability }\end{array}$ & $\begin{array}{c}\text { No } \\
\text { ward } \\
\text { HR }\end{array}$ & $\begin{array}{l}\text { No } \\
\text { ICU } \\
\text { HR }\end{array}$ & $\begin{array}{c}\text { No ICU } \\
\text { or } \\
\text { Ward } \\
\text { HR }\end{array}$ & $\begin{array}{l}\text { Excess } \\
\text { CovID-19 } \\
\text { deaths due } \\
\text { to capacity } \\
\text { breach }\end{array}$ & $\begin{array}{c}\text { Total } \\
\text { Covid- } \\
19 \\
\text { deaths }\end{array}$ & $\begin{array}{c}\% \\
\text { increase } \\
\text { in } \\
\text { COVID- } \\
19 \\
\text { deaths }\end{array}$ \\
\hline Middling & $-20 \%$ & $0 \%$ & $0 \%$ & $0 \%$ & $0 \%$ & 102,573 & 154 & 102,728 \\
\hline Middling & $0 \%$ & $0 \%$ & $0 \%$ & $0 \%$ & $0 \%$ & 102,573 & 154 & 102,728 \\
\hline
\end{tabular}




\begin{tabular}{|c|c|c|c|c|c|c|c|c|}
\hline Middling & $20 \%$ & $0 \%$ & $0 \%$ & $0 \%$ & $0 \%$ & 102,573 & 154 & 102,728 \\
\hline Middling & $0 \%$ & $-20 \%$ & $0 \%$ & $0 \%$ & $0 \%$ & 102,573 & 597 & 103,170 \\
\hline Middling & $0 \%$ & $20 \%$ & $0 \%$ & $0 \%$ & $0 \%$ & 102,573 & 0 & 102,573 \\
\hline Middling & $0 \%$ & $0 \%$ & $-20 \%$ & $0 \%$ & $0 \%$ & 102,573 & 154 & 102,728 \\
\hline Middling & $0 \%$ & $0 \%$ & $20 \%$ & $0 \%$ & $0 \%$ & 102,573 & 154 & 102,728 \\
\hline Middling & $0 \%$ & $0 \%$ & $0 \%$ & $-20 \%$ & $0 \%$ & 102,573 & 130 & 102,703 \\
\hline Middling & $0 \%$ & $0 \%$ & $0 \%$ & $20 \%$ & $0 \%$ & 102,573 & 176 & 102,750 \\
\hline Middling & $0 \%$ & $0 \%$ & $0 \%$ & $0 \%$ & $-20 \%$ & 102,573 & 154 & 102,728 \\
\hline Middling & $0 \%$ & $0 \%$ & $0 \%$ & $0 \%$ & $20 \%$ & 102,573 & 154 & 102,728 \\
\hline Optimistic & $0 \%$ & $0 \%$ & $0 \%$ & $0 \%$ & $0 \%$ & 47,073 & 0 & 47,073 \\
\hline Pessimistic & $-20 \%$ & $0 \%$ & $0 \%$ & $0 \%$ & $0 \%$ & 289,983 & 103,735 & 393,718 \\
\hline Pessimistic & $0 \%$ & $0 \%$ & $0 \%$ & $0 \%$ & $0 \%$ & 289,983 & 73,711 & 363,694 \\
\hline Pessimistic & $20 \%$ & $0 \%$ & $0 \%$ & $0 \%$ & $0 \%$ & 289,983 & 49,178 & 339,161 \\
\hline Pessimistic & $0 \%$ & $-20 \%$ & $0 \%$ & $0 \%$ & $0 \%$ & 289,983 & 76,382 & 366,365 \\
\hline Pessimistic & $0 \%$ & $20 \%$ & $0 \%$ & $0 \%$ & $0 \%$ & 289,983 & 71,253 & 361,236 \\
\hline Pessimistic & $0 \%$ & $0 \%$ & $-20 \%$ & $0 \%$ & $0 \%$ & 289,983 & 66,576 & 356,559 \\
\hline Pessimistic & $0 \%$ & $0 \%$ & $20 \%$ & $0 \%$ & $0 \%$ & 289,983 & 79,764 & 369,747 \\
\hline Pessimistic & $0 \%$ & $0 \%$ & $0 \%$ & $-20 \%$ & $0 \%$ & 289,983 & 73,472 & 363,455 \\
\hline Pessimistic & $0 \%$ & $0 \%$ & $0 \%$ & $20 \%$ & $0 \%$ & 289,983 & 73,926 & 363,909 \\
\hline Pessimistic & $0 \%$ & $0 \%$ & $0 \%$ & $0 \%$ & $-20 \%$ & 289,983 & 72,943 & 362,926 \\
\hline Pessimistic & $0 \%$ & $0 \%$ & $0 \%$ & $0 \%$ & $20 \%$ & 289,983 & 74,197 & 364,180 \\
\hline
\end{tabular}




\section{Figures}

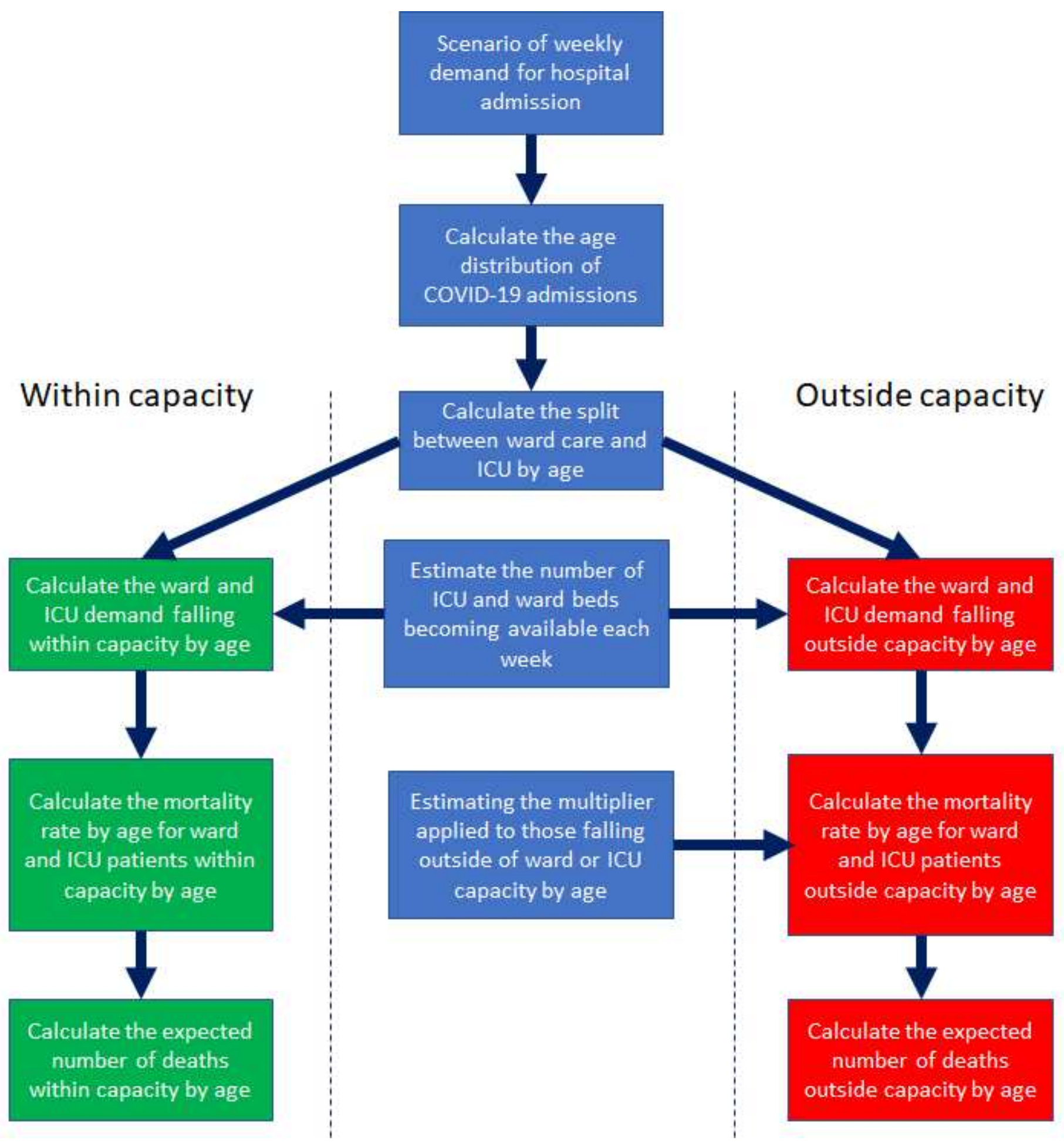

Figure 1

Outline of the structure of the model. 


\section{Admissions demand scenarios}

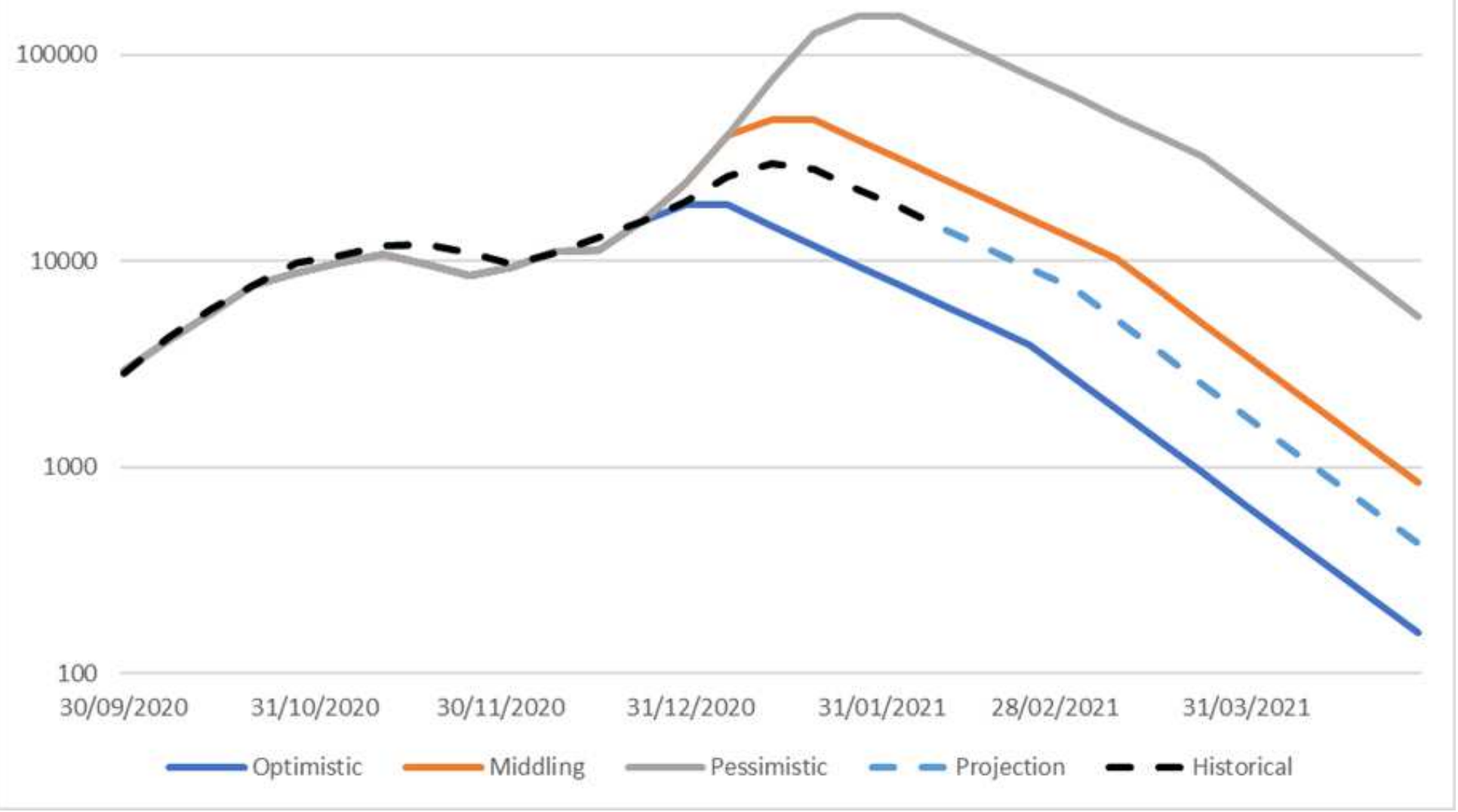

Figure 2

Graph showing three scenarios of projected admission demand from the 9th December 2020. 


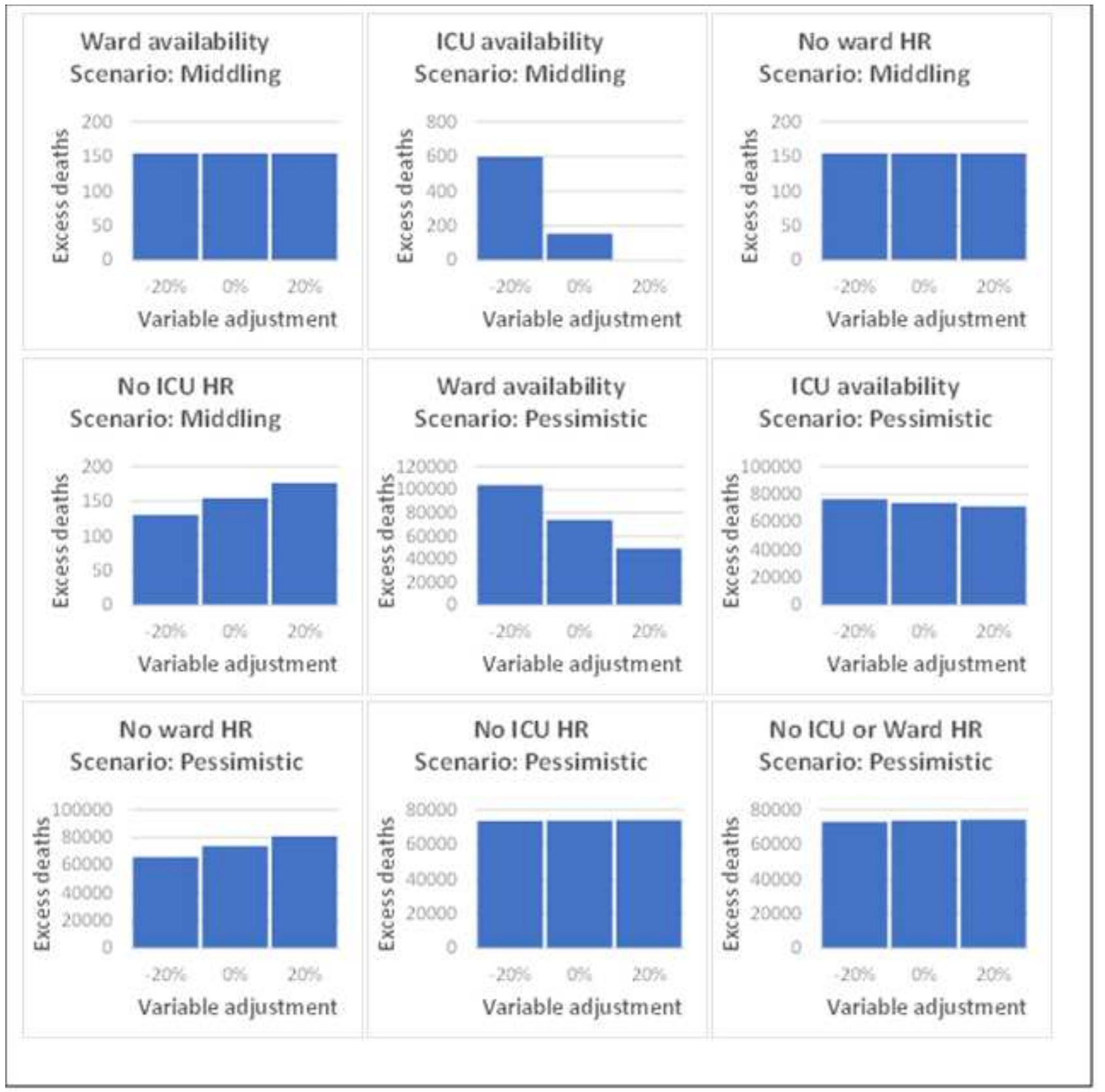

\section{Figure 3}

Results of the sensitivity analysis.

\section{Supplementary Files}

This is a list of supplementary files associated with this preprint. Click to download.

- BMCIFOACOVID1AdditionalFile2Table2AssumptionsExcessMortality.pptx

- COVID19ExceedingCapacityModelMedRxivSubmission210223.xlsx 